\title{
Detecting tidal disruption events of massive black holes in normal galaxies with the Einstein Probe
}

\author{
W. Yuan ${ }^{1}$, S. Komossa ${ }^{1,2}$, C. Zhang ${ }^{1}$, H. Feng ${ }^{3}$, Z.-X. Ling ${ }^{1}$, D.H. \\ Zhao $^{1}$, S.-N. Zhang ${ }^{4}$, J.P. Osborne ${ }^{5}$, P. O'Brien ${ }^{5}$, R. Willingale ${ }^{5}$, \\ J. Lapington ${ }^{5}$ and the Einstein Probe team \\ ${ }^{1}$ Key Laboratory of Space Astronomy and Technology, National Astronomical Observatories, \\ CAS, Beijing, 100012, China \\ email: wmy@nao.cas.cn \\ ${ }^{2}$ Max-Planck-Institut für Radioastronomie, Auf dem Hügel 69, 53121 Bonn, Germany \\ ${ }^{3}$ Department of Engineering Physics, Tsinghua University, Beijing 100084, China \\ ${ }^{4}$ Institute of High Energy Physics, Chinese Academy of Sciences, 100049, Beijing, China \\ ${ }^{5}$ Department of Physics and Astronomy, University of Leicester, Leicester, LE1 7RH, UK
}

\begin{abstract}
Stars are tidally disrupted and accreted when they approach massive black holes (MBHs) closely, producing a flare of electromagnetic radiation. The majority of the (approximately two dozen) tidal disruption events (TDEs) identified so far have been discovered by their luminous, transient X-ray emission. Once TDEs are detected in much larger numbers, in future dedicated transient surveys, a wealth of new applications will become possible. Here, we present the proposed Einstein Probe mission, which is a dedicated time-domain soft X-ray all-sky monitor aiming at detecting X-ray transients including TDEs in large numbers. The mission consists of a wide-field micro-pore Lobster-eye imager $\left(60^{\circ} \times 60^{\circ}\right)$, and is designed to carry out an allsky transient survey at energies of $0.5-4 \mathrm{keV}$. It will also carry a more sensitive telescope for $\mathrm{X}$-ray follow-ups, and will be capable of issuing public transient alerts rapidly. Einstein Probe is expected to revolutionise the field of TDE research by detecting several tens to hundreds of events per year from the early phase of flares, many with long-term, well sampled lightcurves.
\end{abstract}

Keywords. black hole physics, X-rays: bursts, X-rays: general, space vehicles: instruments

\section{Introduction}

Tidal disruption events (TDEs) by massive black holes (MBH) are perhaps the most unique signature of the existence of $\mathrm{MBHs}$ in the cores of otherwise quiescent galactic nuclei (Rees 1988). So far only about two dozen TDE candidates were found, mostly in the declining phase with poorly sampled data, via searches from multi-wavelength surveys (see Komossa 2012 for a review). To detect TDEs in large numbers and to catch the 'smoking gun' at an early phase, it is desirable to monitor a large fraction of the sky with high sensitivity at high cadence, preferably in the soft X-ray band.

\section{The Einstein Probe mission}

Einstein Probe (EP) is a proposed small satellite to detect transients and monitor variable objects in the $0.5-4 \mathrm{keV}$ band, aiming for launch around 2020. EP carries two scientific instruments (Fig. 1) and a fast alert downlink system to trigger followup observations. The primary instrument is a Wide-field X-ray Telescope (WXT) with a FoV of $60^{\circ} \times 60^{\circ}$, which is a lobster-eye type X-ray focusing telescope based on 

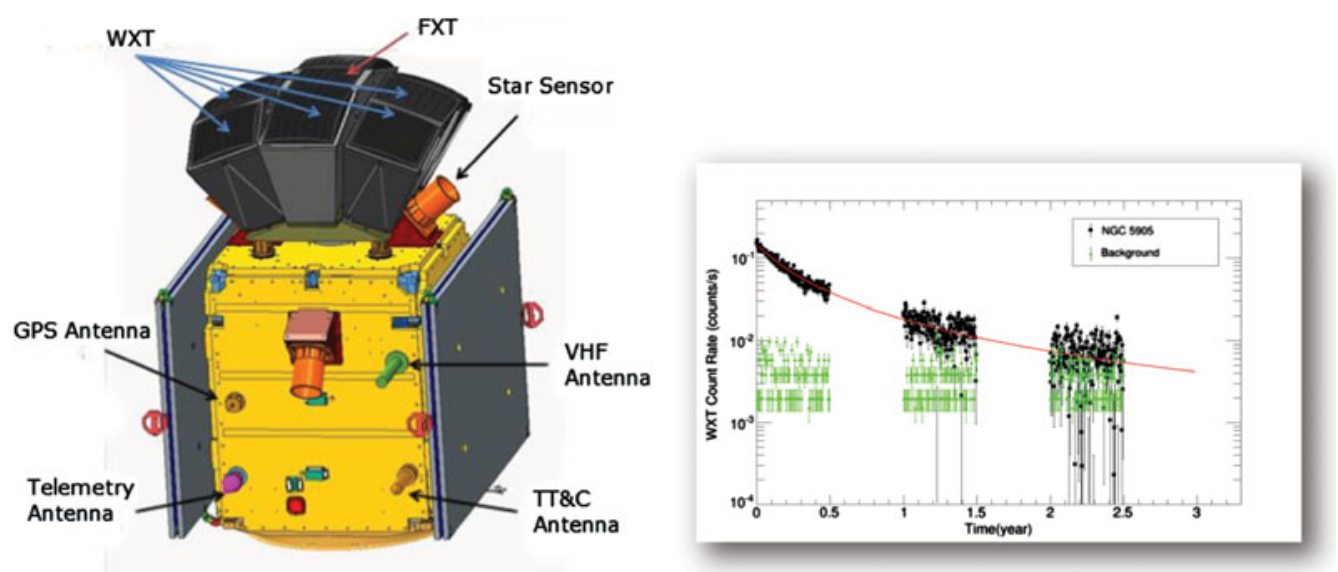

Figure 1. Left: layout of the Einstein Probe satellite. Right: simulated EP/WXT X-ray lightcurve of the TDE found in NGC 5905 (Komossa \& Bade 1999).

Micro-Pore Optics (MPO). In addition, there is a Follow-up X-ray Telescope (FXT) of the same MPO technology, which has a much larger effective area $\left(\sim 60 \mathrm{~cm}^{2}\right)$ but a smaller FoV $\left(1^{\circ} \times 1^{\circ}\right)$ than WXT. EP will offer unprecedentedly high sensitivity and large grasp (see Zhao et al. 2014 for results of simulations of the instrument), which would supersede previous and existing X-ray all-sky monitors. The survey strategy of EP will consist of a series of pointings, and will be able to cover the entire night sky in about 3 orbits ( 90 minutes each orbit). EP has been selected as one of the candidate missions in the Space Science programme of the Chinese Academy of Sciences, and is currently in the 'Advanced Study' phase for mission definition and technology development.

Benefiting from its large field-of-view, high sensitivity, high observing cadence, soft X-ray bandpass, fast alerting and follow-up capability, EP is an ideal mission to systematically search for and characterise TDEs. It is also expected to catch TDEs at an early phase, which is very important for studying the disruption processes, the formation and evolution of accretion discs, the launch of relativistic jets, as well as estimating the mass and spin of the black holes. EP is expected to detect TDEs at the peaks of the $\mathrm{X}$-ray flares out to at least a few hundred Mpc, at an estimated rate from several tens to hundreds per year. Many of them will be caught at the rising phase of the flares, making it possible to observe the events from the very start in multiple wavebands. EP will be able to detect relativistic TEDs with jets similar to Sw J1644+57 (Burrows et al. 2011) out to redshifts $z>1$. Einstein Probe is expected to revolutionise the field of TDE research by detecting and characterising TDEs in large numbers and catching them at the early phase of flares. This will greatly advance our understanding of the demography, formation and evolution of MBHs, as well as the physics of accretion and jet formation.

\section{Acknowledgements}

This work is supported by the Space Science Programme of CAS, Grant XDA04061100.

\section{References}

Burrowset al. 2011, Nature, 476, 421

Komossa, S. 2012, EPJ Web of Conf., 39, id. 02001 
Komossa, S. \& Bade, N. 1999, A\& A, 343, 775

Rees, M. J. 1988, Nature, 33, 523

Zhao, D., et al. 2014, SPIE, Proc. SPIE 9144, Space Telescopes and Instrumentation 2014:

Ultraviolet to Gamma Ray, 91444E (July 24, 2014); doi:10.1117/12.2055434 